\title{
ANXIETY AND STRESS RESPONSE: EFFECTS OF ANXIETY LEVELS AND SEX
}

\author{
Stoyanov Zl., M. Marinov \\ Department of Physiology and Pathophysiology, Medical University - Varna
}

Reviewed by: Assoc. Prof. N. Negrev, MD, PhD

\begin{abstract}
It is an undisputed fact that anxiety may modulate human stress response. However, the existing data on the specificity of the psychophysiological reactivity of high-anxious and low-anxious individuals, and on the presence of sex differences in this context, are controversial. The presented review summarizes recent literary data and analyses some of the neurobiological underpinnings of the sex differences in the association between anxiety and stress response.
\end{abstract}

Keywords: anxiety, stress, sex

Anxiety is a complex psychological construct with various dimensions and manifestations. It contains cognitive, emotional and behavioural components and is accompanied by various physiological changes, which reflect the activity of the autonomic nervous system and neuroendocrine axes $(4,22,21,36,16)$.

In the literature on anxiety and autonomic control there prevail data on increased sympathetic activity, decreased vagal tonic influence on the heart, and reduced autonomic flexibility. Anxiety is associated with increased heart rate (HR), reduced respiratory sinus arrhythmia, increased arterial blood pressure, and higher levels of skin conductance $(4,21,16)$. Some authors find these autonomic changes to be logical, as the mechanisms underlying anxiety are closely connected with the mechanisms of fear and the responses of the type "fight-or-flight" provoked by them (2). It is no accident that many studies put emphasis on the existing two-way close relationship between anxiety and stress. It is assumed that anxiety may be both a natural reaction to a stressor and a part of the complex of the stress response, as well as a post-stressor phenomenon (as in post-traumatic disorder, for example) $(2,24)$. It is also pointed out that anxiety may modulate the stress response and the abilities for coping with stress $(9,33,19)$.

\section{Specificity in the psychophysiological reactivity in low-anxious and high-anxious individuals?}

One of the interesting questions arising in the context of anxiety and autonomic control is whether a psychophysiological stereotyping of the relationship be-

Address for correspondence:

Zlatislav Stoyanov, Dept. of Physiology and Pathophysiology, Medical University, 55 Marin Drinov St, BG-9002 Varna, BULGARIA

E-mail: zsd@mu-varna.bg tween anxiety and autonomic control is possible, and whether there exists specificity in the autonomic reactivity in low-anxious and high-anxious individuals. In a number of publications on the problem, independent of the methodological differences (different measures of anxiety, different stressors and conditions) data are presented that point out that anxiety may moderate psychophysiological reactivity. Reports exist that individuals with high levels of emotional reactivity and trait anxiety are likely to react with a more marked increase of HR and blood pressure (7). In the conditions of cognitive stress Clements and Graham (10) find out that HR reactivity correlates positively with trait anxiety. In the experiments carried out by Gonzalez-Bono et al. (18) and concentrated on the anticipatory autonomic response, a moderating effect of trait anxiety is again presented: high-anxious individuals demonstrate a greater HR reactivity. According to results by Gramer and Saria (19) trait social anxiety exerts a substantial influence on cardiovascular reactivity in active performance situations: high-anxious individuals overall exhibit greater HR reactivity. In a more recent publication, however, which analyses in more detail the cardiovascular effects of the stressor anticipation period, Gramer and Sprintschnik (20) present slightly different data, namely, that high socially anxious individuals overall exhibit lower blood pressure and HR reactivity. That corresponds with the earlier data by Wilken et al. (44), who also report of poorer reactivity in high-anxious individuals. Similar are the data from the population-based study of Young et al. (47): individuals with high trait anxiety demonstrated reduced cardiovascular reactivity whereas individuals with trait anxiety demonstrated increased reactivity. It is important to draw attention to the fact that in that study the group of high anxiety subjects consisted predominantly of women. In contrast to the above, in their study Mauss et al. (28) find out that the psychophysiological responses of high-anxious individuals 
during anticipatory anxiety are similar to those of low-anxious individuals. In accordance with them is the opinion of Barret and Armony (2) that individual differences in trait anxiety do not affect the autonomic responses associated with a mental task.

Not so definitive are also some data from the studies analyzing the neuroendocrine activity. Some authors report that in anxiety disorder patients exaggerated acute neuroendocrine responses to psychosocial stressors are observed (8). Other studies point out, however, that individuals with high levels of TA have reduced neuroendocrine reactivity to stressors. Jezova et al. (22) established that subjects with high trait anxiety exhibit lower plasma levels of adrenocorticotropic hormone (ACTH) and cortisol, and lower stress-induced activation of epinephrine and norepinephrine secretion. There also exist mixed results: Gerra et al. (17) report that after a psychological stress the concentrations of norepinephrine, growth hormone and testosterone increase significantly only in anxious individuals, but substantial changes in the concentrations of ACTH, cortisol and epinephrine are absent. Takahashi et al. (36) find out significant positive correlation between trait anxiety, autonomic reactivity and basal cortisol levels at rest, but not in stress conditions.

\section{Sex differences in the association between anxiety and psychophysiological responses to stress}

The reasons for the ambiguous and conflicting results may be different, but it is not impossible for it to be due to the fact that the role of the sex has not been analysed in detail (18): either mixed groups have been studied and the data for both sexes have been interpreted together, or the conclusions have been reached over observation of only men $(17,21,36)$, or only women $(18,33,19,20)$.

Concentrating on the role of the sex is well-founded not only because of the different levels of anxiety reported in men and women - many authors share the view that women are more anxious than men $(3,31,15,26,11)$, but also because of the existing data for a different pattern of the association between emotions and physiological parameters in men and women (30). There are reports that anxious men show higher reactivity of the diastolic blood pressure and cortisol secretion while anxious women show more pronounced changes in the HR (13). Under laboratory stress (public speech) Carrillo et al. (9) find out in men significant positive correlation between state anxiety and peripheral pulse volume, but not between state anxiety and HR. In contrast to that, in women the same authors observe positive correlation between anxiety and reactivity of HR. The differentiated by sex analysis of our experimental results suggests the existence of typical cardiovascular responses to mental stress of low-anxious and high-anxious individuals (27), however the parameters of this stereotyping are reciprocal for men and women, which may mask as a whole the effect of the anxiety on the cardiovascular response to stress. So we support the view of Naveteur and Freixa-i-Baque (32) that anxiety may moderate the psychophysiological responses to stressful situations, but unlike them, we believe that in this aspect there exist sex differences. We will also add that our results do not correlate with the data of Takai et al. (37) as well, about the absence of sex differences in the sympathetic-adrenomedullar activity during stress in high-anxious and low-anxious individuals. According to the same authors however, during stress the cortisol levels in high-anxious women are significantly lower that those in high-anxious men.

In several of the researches that study the correlations between anxiety and psychophysiological reactivity to stress in women $(33,19,20)$, the established type of reaction in high-anxious and low-anxious individuals is interpreted within the framework of active coping and energization and is connected with the subjective evaluations of the individuals as to the gravity of the task, the efforts invested and the likelihood of success, their perception of stress and the means to cope with it. There do not exist, however, enough comparable data for men.

When clarifying the role of the sex in the relationship between anxiety and stress, there should also be taken into account the existence of sex differences in stress reactivity. Most (but not all) of the data present in literature outline a tendency to a more acute stress response of the sympathetic-adrenal mechanisms in men when compared to women $(25,23)$. A different pattern of brain activity in men and women in stress conditions has also been established. Mental stress in men is associated with activation of the right prefrontal cortex and reduced activity in the left orbitofrontal cortex, while in women structures of the limbic system become active, like the ventral striatum, putamen, insula and cyngular cortex (43). At the same time the dominating right-sided prefrontal activity during stress in men correlates significantly and with changes in the physiological parameters like the cortisol response, but the limbic activation in women correlates quite weakly with cortisol. In addition to this, in women correlation is established between anxiety related personality traits and regional brain volume in the left anterior prefrontal cortex smaller brain volume in the left anterior prefrontal cortex underlies the basis for higher anxiety-related traits (46).

The question of the sex differences in the association between anxiety and psychophysiological responses to stress becomes even more topical due to the fact that in recent years there are attempts to isolate two different models of stress response: "fight-or-flight" and "tend-and-befriend" $(39,40,38)$. As we pointed above, data from our study showed that high-anxious men are characterized by a more strongly expressed cardiovascular reactivity under stress, while high-anxious women are characterized with less expressed response of HR and vascular tone. The "logic" of the established sex differences in the correlation between trait anxiety and cardiovascular reactivity could be partly explained with the mentioned two different models of stress response: "fight-or-flight", accepted for a "male" type, and "tend-and-befriend", accepted for a "female" type $(39,40,38)$. It is known that non-pathological anxiety could be a useful factor for adequate organization of the behavior 
under potential threat for the individual $(34,6)$. According to their evolutionary role (hunters and warriors) men should have optimally secured reactions of the "fight-or-flight" type. In that context it seems logical that in men there exists a marked positive correlation (or maybe potentiation) between anxiety and autonomic stress response. It is possible for androgens to act as mediators in this association. It is known that testosterone levels (in principle higher in men) increase significantly with acute stress (39). There are also data that make us think that the prenatal organizational effects of testosterone on the brain could be reflected in anxiety as well $(14,1)$.

The stress response of "tend-and-befriend", accepted as more typical for women, reflects the evolutionary role of women to ensure the survival of the offspring and for that purpose it is expedient for them for preserve themselves through other behavioral strategies $(39,40,38)$. The authors cited suggest that female responses to stress may build on attachment/caregiving processes that downregulate sympathetic and hypothalamic-pituitary-adrenocortical responses to stress. Oxytocin is ascribed a controlling effect in such situations $(29,39,38)$, and there are data that it increases the excitability of vagal neurones $(12,35)$ and decreases sympathetic activity (41).

On that background it can be speculated that anxiety "serves" in different ways the stress response in men and women. The positive correlation between trait anxiety and cardiovascular reactivity to stress in men suggests that in male sex the higher anxiety may act as a catalyst of the autonomic parameters of stress response. If we accept that the weaker cardiovascular reactivity observed by us in high-anxious women is a manifestation of downregulation of the autonomic mechanisms, then we should suppose that in women the higher anxiety compensates to some extent the tendency for weaker sympathetic-adrenal reaction and ensures specific adequacy of the stress response on principle. Here, however, a certain discrepancy arises. The mechanisms of downregulation in "tend-and-befriend" are accepted to be oxytocin-mediated and moderated by the estrogens and the endogenous opioids $(45,39)$, but these hormones and peptides are known to have anxiolytic properties $(45,39,42)$. Probably it has to do with a more complex reaction of the brain regions (cortex, subcortical nuclei, diencephalon) whose interaction ensures the brain integration of mental and physiological (somatic) functions.

The cohesion of some data from studies of the brain correlates of anxiety and stress gives reason to think that the sex differences in the association between anxiety and psychophysiological reactivity to stress reflect the existence of special sex features of the brain integration of cognitive processes, emotions and autonomic control.

\section{REFERENCES:}

1. Alpers, G., W., A. B. M. Gerdes. Show of hands: first evidence for an influence of prenatal testosterone on anxiety disorders. In: Psychologie und
Gehirn. E. Wascher, M. Falkenstein, G. Rinkenauer, M. Grosjean, Eds. 2007, 96. http://www.ifado.de/pg2007/Tagung_Abstracts.pdf

2. Barret, J., J. Armony. The influence of trait anxiety on autonomic response and cognitive performance during an anticipatory anxiety task.-Depress. Anxiety, 23, 2006, 210-219.

3. Ben-Zur, H., M. Zeidner. Sex differences in anxiety, curiosity, and anger: A cross-cultural study.-Sex Roles, 19, 1988, 335-347.

4. Bernston, G. G., J. T. Cacioppo. Heart rate variability: stress and psychiatric conditions. In: Dynamic Electrocardiography. M. Malik, A. J. Camm, Eds., Elmsford, New York, Blackwell Futura Publishing, 2004, 57-64.

5. Bersenev, V., G. Guba, O. Pyatak. Handbook of Clinical Neurovegetology, Kiev, Zdorovya, 1990, 191-194. (in Russian)

6. Brown, L. A., J. B. Doan, N. C. McKenzie, S. A. Cooper. Anxiety-mediated gait adaptations reduce errors of obstacle negotiation among younger and older adults: Implications for fall risk.-Gait Posture, 24, 2006, 418-423.

7. Carels, R. A., J. A. Blumenthal, A. Sherwood. Emotional responsivity during daily life: relationship to psychosocial functioning and ambulatory blood pressure.-Int. J. Psychophysiol., 36, 2000, 25-33.

8. Carrasco, G. A., L. D. Van de Kar. Neuroendocrine pharmacology of stress. Eur. J. Pharmacol., 463, 2003, 235- 272.

9. Carrillo, E., L. Moya-Albiol, E. Gonzalez-Bono, A. Salvador, J. Ricarte, J. Gomez-Amor. Gender differences in cardiovascular and electrodermal responses to public speaking task: the role of anxiety and mood states.-Int. J.

Psychophysiol., 42, 2001, 253-264.

10. Clements, K., T. Graham. Life event exposure, physiological reactivity, and psychological strain.-J. Behav. Med., 23, 2000, 73-94.

11. Dickie, E. W., J. L. Armony. Amygdala response to unattended fearful faces: Interaction between sex and trait anxiety.-Psychiat. Res-Neuroim., 162, 2008, 51-57.

12. Dreifuss, J. J., M. Dubois-Dauphin, H. Widmer, M. Raggenbass. Electrophysiology of oxytocin actions on central neurons.-Ann. NY Acad. Sci., 652, 1992, 46-57.

13. Earle, T. L., W. Linden, J. Weinberg. Differential effects of harassment on cardiovascular and salivary cortisol stress reactivity and recovery in women and men.-J. Psychosom. Res., 46, 1999, 125-141.

14. Evardone, M., G. M. Alexander. Anxiety, sex-linked behaviors, and digit ratios (2D:4D).-Arch. Sex. Behav., 2007, 10.1007/s10508-007-9260-6 (Epub ahead of print).

15. Feingold, A. Gender differences in personality: A meta-analysis.-Psychological Bulletin, 116, 1994, 429-456.

16. Friedman, B. H. An autonomic flexibility-neurovisceral integration model of anxiety and cardiac vagal tone.-Biol. Psychol., 74, 2007, 185-199.

17. Gerra, G., A. Zaimovic., U. Zambelli, M. Timpano, N. Reali, S. Bernasconi et al. 
Neuroendocrine Responses to Psychological Stress in Adolescents with Anxiety Disorder.-Neuropsychobiology, 42, 2000, 82-92.

18. Gonzalez-Bono, E., L. Moya-Albiol, A. Salvador, E. Carrillo, J. Ricarte, J.

Gomez-Amor. Anticipatory autonomic response to a public speaking task in women. The role of trait anxiety.-Biol. Psychol., 60, 2002, 37-49.

19. Gramer, M., K. Saria. Effects of social anxiety and evaluative threat on cardiovascular responses to active performance situations.-Biol. Psychol., 74, 2007, 67-74.

20. Gramer, M., E. Sprintschnik. Social anxiety and cardiovascular responses to an evaluative speaking task: The role of stressor anticipation.-Pers. Indiv. Differ., 44, 2008, 371-381.

21. Hofmann, S.G., D. A. Moscovitch, B. T. Litz, H. J. Kim, L. L. Davis, D. A. Pizzagalli. The worried mind: autonomic and prefrontal activation during worrying.-Emotion, 5, 2005, 464-475.

22. Jezova, D., A. Makatsori, R. Duncko, F. Moncek, M. Jakubek. High trait anxiety in healthy subjects is associated with low neuroendocrine activity during psychosocial stress.-Prog. Neuro-psychoph., 28, 2004, 1331-1336.

23. Kajantie, E., D. I. Phillips. The effects of sex and hormonal status on the physiological response to acute psychosocial stress.-Psychoneuroendocrino., 31, 2006, 151-178

24. Keane, T. M., K. L. Taylor, W. E. Penk. Differentiating Post-Traumatic Stress Disorder (PTSD) from Major Depression (MDD) and Generalized Anxiety Disorder (GAD).-J. Anxiety Disord., 11, 1997, 317-328.

25. Kudielka, B. M., C. Kirschbaum. Sex differences in HPA axis responses to stress: a review.-Biol. Psychol., 69, 2005, 113-132.

26. Lindová, J., M. Hrusková, M. Pivonková, A. Kubena, J. Fleger. Digit ratio (2D:4D) and Cattell's personality traits.-Eur. J. Personality, 2007, DOI: $10.1002 /$ per.664.

27. Marinov, M., Z. Stoyanov, I. Boncheva, I. Vartanyan, T. Chernigovskaya. Trait anxiety and peripheral vascular response to mental stress - sex differences.-Int. J. Psychophysiol., 2008 (in press).

28. Mauss, I. B., F. H. Wilhelm, J. J. Gross. Autonomic recovery and habituation in social anxiety.-Psychophysiology, 40, 2003, 648-653.

29. McCarthy, M. M. Estrogen modulation of oxytocin and its relation to behavior. In: Oxytocin: Cellular and molecular approaches in medicine and research. R. Ivell, J. Russell, Eds. New York, Plenum Press, 1995, 235-242.

30. Moya-Albiol, L., A. Salvador, R. Costa, S. Martinez-Sanchis, E. González-Bono, J. Ricarte et al. Psychophysiological responses to the Stroop task after a maximal cycle ergometry in elite sportsmen and physically active subjects.-Int. J. Psychophysiol., 40, 2001, 47-59.

31. Nakazato, K., Y. Shimonaka. The Japanese State-Trait Anxiety Inventory: age and sex differences.-Percept. Motor Skill., 69, 1989, 611-617.
32. Naveteur, J., E. Freixa-i-Baque. Individual differences in electrodermal activity as a function of subjects' anxiety.-Pers. Indiv. Differ., 8, 1987, 615-626.

33. Naveteur, J., S. Buisine, J. H. Gruzelier. The influence of anxiety on electrodermal responses to distractors.-Int. J. Psychophysiol., 56, 2005, 261-269.

34. Nesse, R. M. What Darwinian Medicine Offers Psychiatry. In: Evolutionary Medicine. W. R. Trevathan, J. J. McKenna, E. O. Smith. Eds. New York, Oxford University Press, 1999, 351-373.

35. Raggenbass, M., J. J. Dreifuss. Mechanism of action of oxytocin in rat vagal neurones: induction of a sustained sodium-dependent current.-J. Physiology, 457, 1992, 131-142.

36. Takahashi, T., K. Ikeda, M. Ishikawa, N. Kitamura, T. Tsukasaki, D. Nakama et al. Anxiety, reactivity, and social stress-induced cortisol elevation in humans.-Neuroendocrinol. Lett., 26, 2005, 351-354.

37. Takai, N., M. Yamaguchi, T. Aragaki, K. Eto, K. Uchihashi, Y. Nishikawa. Gender-specific differences in salivary biomarker responses to acute psychological stress.- Ann. NY Acad. Sci., 1098, 2007, 510-515.

38. Taylor, S. E. Tend and befriend: biobehavioral bases of affiliation under stress.-Curr. Dir. Psychol. Sci., 15, 2006, 273-277.

39. Taylor, S. E., L. C. Klein, B. P. Lewis, T. L. Gruenewald, R. A. R. Gurung, J. A.

Updegraff. Biobehavioral responses to stress in females: tend-and-befriend, not fight-or-flight.-Psychol. Rev., 107, 2000, 411-429.

40. Turton, S., C. Campbell. Tend and befriend versus fight or flight: gender differences in behavioral response to stress among university students.-J. Appl. Biobehav. Res., 10, 2005, 209-232.

41. Uvnas-Moberg, K. Oxytocin linked antistress effects - the relaxation and growth response.-Acta Psychol. Scand., Suppl. 640, 1997, 38-42.

42. Walf, A. A., C. A. Frye. A review and update of mechanisms of estrogen in the hippocampus and amygdala for anxiety and depression behavior.-Neuropsychopharmacol., 31, 2006, 1097-1111.

43. Wang, J., M. Korczykowski, H. Rao, Y. Fan, J. Pluta, R. C. Gur, et al. Gender difference in neural response to psychological stress.-Soc. Cogn. Affect. Neurosci., 2, 2007, 227-239.

44. Wilken, J. A., B. D. Smith, K. Tola, M. Mann. Trait anxiety and prior exposure to non-stressful stimuli: effects on psychophysiological arousal and anxiety.- Int. J. Psychophysiol., 37, 2000, 233-242.

45. Windle, R. J., N. Shanks, S. L. Lightman, C. D. Ingram. Central oxytocin administration reduces stress-induced corticosterone release and anxiety behavior in rats.-Endocrinology, 138, 1997, 2829-2834.

46. Yamasue, H., O. Abe, M. Suga, H. Yamada, H. Inoue, M. Tochigi, et al. Gender-common and -specific neuroanatomical basis of human anxiety-related personality traits.-Cereb. Cortex, 18, 2008, 46-52

47. Young, E. A., R. M. Nesse, A. Weder, S. Julius. Anxiety and cardiovascular reactivity in the Tecumseh population.-J. Hypertens., 16, 1998, 1727-1733. 